\title{
Rollover Propensity Evaluation of an SUV Equipped with a TRW VSC System
}

\author{
Ali Y. Ungoren, Huei Peng \\ University of Michigan \\ Danny R. Milot \\ TRW Inc.
}

Copyright (? 2000 Society of Automotive Engineers, Inc.

\begin{abstract}
In this paper, a simulation-based dynamic rollover evaluation procedure is described. This work is based on the worst-case methodology developed at the University of Michigan, and is the result of a collaborated research project between the University of Michigan and TRW Inc. The target vehicle studied in this paper is a large production volume SUV. This vehicle is equipped with a production-intent TRW Vehicle Stability Control (VSC) system. The main goals of this paper are to (i) study the rollover propensity of this SUV, as influenced by vehicle and environment parameters such as vehicle speed, road condition, etc.; and (ii) investigate whether, and by how much, does the VSC system influence the rollover propensity of this SUV. The modeling, evaluation procedure, and preliminary evaluation results are reported.
\end{abstract}

\section{INTRODUCTION}

The growing popularity of Sports Utility Vehicles (SUV) in the last decade together with their higher rollover probability has necessitated a closer look at regulations to reduce fatalities in their rollover crashes. NHTSA reported that there were 10,857 rollover deaths in cars, vans and trucks last year, up from 9,771 in 1998, which seems to be closely related to the increased population of SUVs and light trucks. Recently (May 2000), the National Highway Traffic Safety Administration (NHTSA) announced its plan to include a vehicle measure of rollover resistance as an addition to the 2001 New Car Assessment Program (NCAP). NHTSA determined that a vehicle's "Static Stability Factor" (SSF) is the most reliable indicator of rollover risk of un-tripped singlevehicle crashes. NHTSA expects that its new rollover information program will motivate manufacturers to produce safer, more stable vehicles. Currently, the U.S. Congress is temporarily holding the publication of this new "star rating" system. Obviously, the rollover propensity of SUV is an important issue that has caught the attention of consumers, government agencies, car companies, and major suppliers.
For assessing the on-road, untripped rollover propensity of a vehicle, there were predominantly two types of metrics proposed in the literature. The NHTSA dynamic test program uses a set of rollover evaluation maneuvers and examines the severity and frequency of vehicle Two-Wheel-Lift (TWL) under these maneuvers. The Consumer Union's double lane change driving test also falls into this "dynamic testing" category. The "static" type of rollover metrics, on the other hand, is usually based on the simple measurement of vehicle parameters related to its roll behavior. For example, Static Stability Factor, Tilt Table Angle, Tilt Table Ratio, Critical Sliding Velocity and Side Pull Ratio all fall into this category. In general, static testing results may not completely incorporate the effects of controllers (e.g., vehicle stability control systems and $A B S$ ), transient tire behavior, and so forth.

In literature it has been shown that a properly designed active control system could reduce the risk of rollover. Four-wheel steering (4WS), active suspensions, passive suspensions accompanied by anti-roll bars, suspensions with variable stiffness and differential braking have all been studied for their effect on roll control. For heavy articulated trucks with multiple axles, the steering of tractor wheels was shown to improve lateral stability (Furleigh et al. 1988). Steering the rear axles of the tractor proportional to the front axles, with a proportionality constant dependent on the forward speed, reduced the lateral acceleration of the trailer at high-speed obstacle maneuvers. Cech et al. (2000) proposed two configurations--anti-roll and active roll suspensions for better cornering performance. The Antiroll design uses hydraulically activated anti-roll bars implemented with passive suspensions. The latter offers anti-roll control as well as independent control of each suspension. Another approach for reducing the roll angle is to vary the suspension stiffness without adding any energy to the system (Constantine et al. 1994). Palkovics et al. (1999) proposed a Roll-Over Prevention $\left(\mathrm{ROP}^{\circledR}\right)$ system for commercial vehicles. Wheel speed and lateral acceleration are used to estimate the wheel 
lift-off. Full (left and right hand sides) braking of the vehicle is activated if a wheel lift-off is detected. In another study, Wielenga (1999) proposed an AntiRollover Braking $\left(\mathrm{ARB}^{\mathrm{TM}}\right)$ system, which utilizes differential braking (braking on one side of the vehicle tires). If an impending rollover is predicted, either due to the existence of high lateral acceleration or the direct sensor detection of wheel lift-off, differential braking is applied to slow down the vehicle as well as to reduce the lateral acceleration.

The development of these advanced roll control systems are likely to accelerate in the coming decade. A forwardlooking government-defined regulation/standard should incorporate a well-designed dynamic test procedure to obtain a more accurate assessment of vehicle rollover propensity, especially for those vehicles equipped with advanced control systems.

\section{VEHICLE MODELS}

Upon the request of the OEM, we are not going to mention the model name of the target SUV studied in this paper. Rather, it will be referred to as "Vehicle A" in the remainder of this paper. For comparison purposes, we will provide un-compensated (no VSC control) vehicle simulation results of a benchmark vehicle-a 1998 Jeep Cherokee. The benchmark vehicle will be referred to as "Vehicle B" from now on. The mathematical models for these two vehicles are described in the following:

\section{Vehicle A:}

- The vehicle dynamics model was created using the AutoSim software by Mechanical Simulation Corporation (MSC). The model was created for TRW as an extension to MSC's CarSim product.

- The model has 14 DOF (6 DOF for the sprung mass, 2DOF for each of the axles, and 1 DOF for each of the wheels).

- The model also includes a production-intent VSC control algorithm incorporated as a C-code module. In addition to the control algorithm, a production intent hydraulic control unit model is used to provide the active brake torque control. A lumped parameter powertrain model is also used for interaction with the VSC system for understeer control.

\section{Vehicle B:}

- The mathematical model is created using the TruckSim software (also the product of MSC), and is based on vehicle parameters published by the Vehicle Research and Testing Center of the NHTSA [Salaani et al. 1999]. This TruckSim model was verified against VRTC published test results (Chen and Peng 1999) at 2 different vehicle speeds with steering and braking inputs that generate lateral acceleration as high as $0.6 \mathrm{~g}$.
- The model also has 14 DOF (identical to those of Vehicle A model).

Both models include nonlinear models for tires, suspension springs, shocks, brakes, etc. The models are capable of predicting 3D forces and motions of vehicles in response to throttle (Vehicle $A$ only), braking and steering inputs. The Vehicle A model also includes a powertrain model developed by TRW.

\section{WORST-CASE EVALUATION PROCEDURE}

Over the last two decades, computer-aided engineering has made tremendous progress in the automotive industry. One of the most noticeable trends is the growing popularity of computer-based simulation models to replace prototypes or early production vehicles. These models are now used extensively in crashworthiness, NVH, dynamic testing, etc. The worstcase evaluation method developed at the University of Michigan (Ma and Peng 1999) aims to use optimization techniques to identify weaknesses of vehicles and the worst-case scenarios (steering input, braking input, operational parameters, environmental parameters) that can be iteratively figured out by trial-and-error or by intuitive reasoning. This simulation-based approach provides a cheaper alternative to the existing test-matrix based approach. This is because test engineers' experience may be quite valuable for simple singleinput-single-output vehicle response. However, as the (vehicle) dynamic model becomes very complex and/or nonlinear, finding the weaknesses of a vehicle design becomes strenuous and more trial-based even for experienced engineers. Therefore, a standard practice is to define a richer and richer test matrix, in hope that by testing enough samples, the relative performance of a vehicle design can be reasonably understood. Yet, the essence of a comprehensive evaluation of the "safety" aspect of a new vehicle before its production enforces the use of more effective means to identify its "worstcase" behavior, which may or may not be captured in the test matrix. Therefore, the simulation-based approach seems to be more viable compared with the test-matrix based approach.

To this end, the worst-case evaluation methodology, introduced in this paper, proves to be an efficient method. This method provides a systematic analysis of all the available data about the dynamic system and displays the conditions under which the system fails to meet the desired requirements. In the following section, we give the mathematical model of the dynamic system, which is an SUV in our case. By using the worst-case methodology, it is possible to determine the worst inputs (or more precisely, disturbance inputs, $w(t)$ ) and the optimal control inputs $(\mathrm{u}(\mathrm{t}))$ opposing the disturbance. In this study, we will be focusing on the identification of the worst disturbances $(\mathrm{w}(\mathrm{t}))$ to the vehicle, which are assumed to include hand-wheel steering angle and brake pedal force. The worst-case disturbance inputs aim to minimize, while the control inputs try to maximize "safety". In this paper, we will focus only on the vehicle 
rollover problem. In other words, $\mathrm{w}(\mathrm{t})$ tries to maximize vehicle roll angle. The control input $u(t)$, when it exists, is assumed to be the brake pressure command provided by the VSC system.

In the mathematical form, the worst-case problem is defined as follows. The dynamic system of interest is assumed to be described by the nonlinear equation $\dot{x}=f(x, w, u, t) \quad$ (Figure 1), where $\mathrm{w}(\mathrm{t})$ and $\mathrm{u}(\mathrm{t})$ are the "bad" and "good" (disturbance and control) inputs. The state vector $x$ includes all vehicle dynamic variables, including, for example, sprung mass roll angle, vehicle side slip angle, etc.

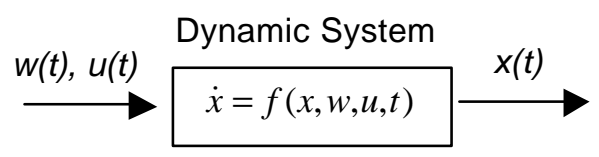

Figure 1 The abstract nonlinear system

The objective of the disturbance input is to maximize a performance index in the following general form:

$$
J=\phi\left[x\left(t_{f}\right)\right]+\int_{t_{0}}^{t_{f}} L(x, u, v, w, t) d t
$$

where the functions $\phi(x)$ and $L(x, u, v, w, t)$ are defined for different "safety" purposes. For example, for this paper, since the focus is on vehicle rollovers, these two functions will include heavy penalty weighting on vehicle roll angle and/or roll angle rates. Studies on the solutions of the above optimization problem date back to as early as the $17^{\text {th }}$ century. If the dynamic system model is nonlinear, finding an analytic solution to the above problem is not trivial. Therefore, numerical methods are usually adopted. The worst-case methodology reduces the above problem into a two-point boundary value problem (TPBVP), and solves the resulting equations iteratively to identify the optimal value for the performance index, and the associated $w(t)$ signal. Through this process, in theory we can also solve the optimal value for $u(t)$, which could be viewed as the "model" for a good control signal. However, in this paper, we will focus on the worst-case disturbance generation only, in other words, $u(t)$ will not be optimized. Instead, it is assumed to be either nonexistent (for a vehicle with no-control) or generated by a pre-determined control algorithm.

As in all iterative numerical methods, achieving the global optimal solution is usually not guaranteed, and the quality of the final solution highly depends on the initial guesses (IG, of $w(t))$. A successful numerical scheme usually includes two kinds of IG generation mechanisms: Initial guesses that are believed to be close to a very good local optimal point, based on which, a search in a small neighborhood usually yields fruitful results (the socalled shot-gun IG), and a systematic, grid-search type of IG which may or may not be followed by a neighborhood search (the so called machine-gun IG).

In this paper, initial guesses are obtained by three different ways: the standard maneuvers from literature (e.g., rollover evaluation maneuvers used by VRTC), intuition ("common-sense" maneuvers suggested by experienced engineers) or by iterative dynamic programming (IDP, Luus 2000). The IDP approach is a little more systematic compared to the other methods because the initial guesses generated by this method are more likely to converge to the global optimal solution, or at least a very good local optimal point. Therefore, we will be utilizing IDP as a major tool for the initial guess selection and TPBVP approach for the worst-case solution. The algorithm for the overall numerical method is outlined below.

\section{Outline of the Worst-case Algorithm:}

1. Define cost function with proper weighting factors.

2. Select vehicle operational parameters (initial speed, weight, etc.) and environmental parameters (e.g. road friction) and input ranges (e.g., steering wheel angle limited to \pm 100 degrees, brake pedal force limited between 0 and 150lbf, etc.)

3. Divide the problem horizon of $\left[0, t_{f}\right]$ into $P$ time stages.

4. Pick $N$ grid points for each of the inputs (steering and braking) in the range specified in step 2.

5. Start from $(P-1)^{\text {th }}$ time point and evaluate the performance index at the time interval from $(\mathrm{P}-1)$ to $t_{f}$ for each of the $\mathrm{N}$ grid points picked for that input. Save the input for the optimal cost function $J$ at this time interval and step backward to the preceding time point $(P-2)^{\text {th }}$ to evaluate the optimal $J$ of the input from $(P-2)^{\text {th }}$ to $\hbar$. Repeat the evaluation of the optimal $J$ at each of the time intervals in the same manner, by stepping backward each time to the previous time point until the overall optimal $J$ of the input is determined for the entire time interval from 0 to $t_{f}$.

6. Reduce the allowed input range.

7. Refine IG (go to step 4), or go to step 8 below.

The seven steps described above help to find a good initial guess. Meanwhile, standard testing maneuvers such as J-turn, brake in a J-turn, fishhook maneuvers and random inputs satisfying the input constraints can also be used as initial guesses. Finally, worst-case solutions identified for a vehicle under different operating conditions (speed, road condition) or from different but similar vehicles can also serve as candidates of initial guesses.

\section{Searching Process:}

8. Use the initial guess obtained above to simulate the dynamic model forward in time from $t=0$ to $t=t_{f}$. 
9. Save the state histories, evaluate necessary gradient information.

10. Update the identified disturbance signal according to the gradient information.

11. Continue iteration (go to step 6) until the desired solution is obtained.

Based on the algorithm defined, a worst-case evaluation program is created by using the MATLAB software package from the Mathworks Inc. A schematic diagram showing how this evaluation method works is given in Figure 2 with its user interface shown in Figure 3, and the animation example shown in Figure 4. The core of this simulation package is the dynamic model of the vehicle with proper input signals defined and all its states and state derivatives available as outputs. The optimization routine then searches for the worst-case inputs in an iterative manner. Equations of the vehicle dynamics need not be known exactly and/or be shown in state equation form. However, if they are given, the speed of convergence to the solutions will improve considerably. Depending on the definition of the performance index $(\mathcal{J})$, worst-case scenarios can be determined for different aspects of the vehicle performance (roll, yaw stability).

\section{SIMULATION RESULTS}

Worst Case Maneuvers: The worst-case methodology described in the previous section is used to determine hand-wheel steering and brake pedal force combinations for SUV rollover propensity assessment. With appropriate saturation bounds such as steering angle and steering rate imposed, these input signals then simulate the worst-possible handling/braking inputs from human drivers. (in terms of rollover).

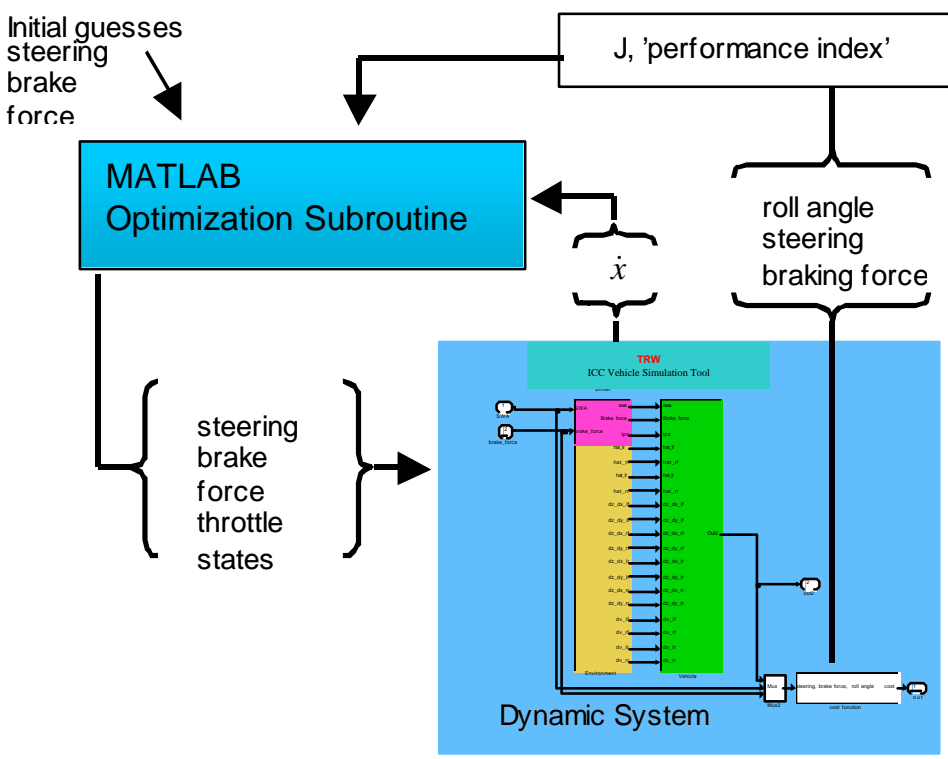

Figure 2: Architecture of the worst-case evaluation program

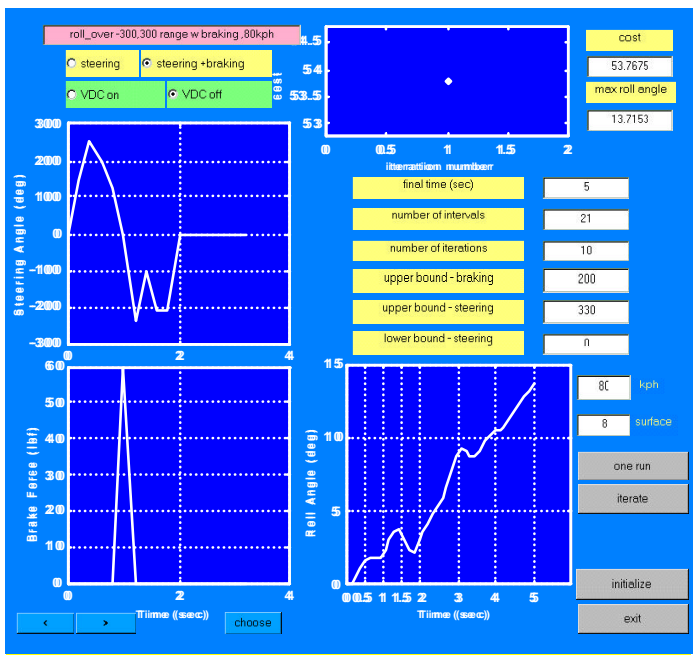

Figure 3: User interface of the worst-case evaluation program.

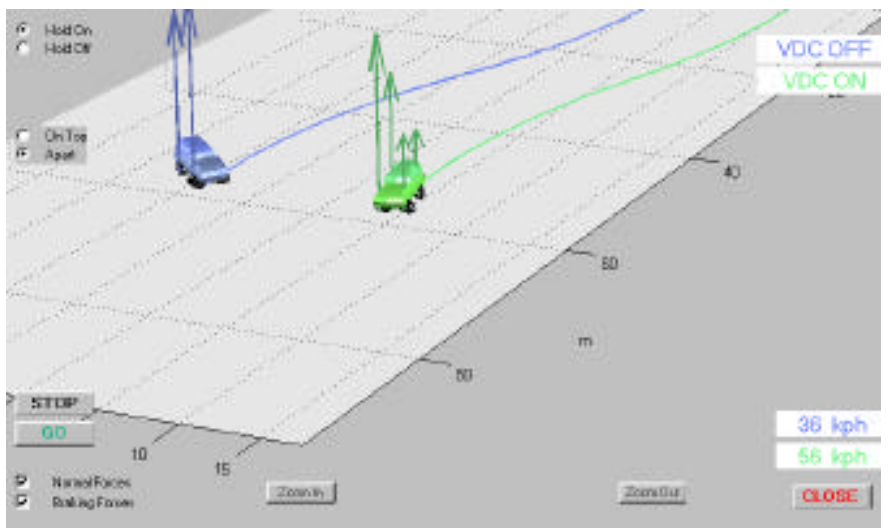

Figure 4: A screen shot from the VSC-on \& VSC-off comparison animation, where arrows indicate tire normal forces.

Standard Maneuvers: The dynamic testing procedure used in the NHTSA Phase II study included several maneuvers that were intuitively selected to represent maneuvers preceding rollover accidents. We will adopt several of these maneuvers, including the J-Turn, fishhook \#1 and \#2 in our simulations (see appendix for details of these maneuvers). The steering amplitudes of these maneuvers are sometimes scaled to make the comparison meaningful.

\section{SIMULATION MATRIX}

Case Studies:

1. Under the same steering angle and brake pedal force limit (200 lbf for pedal force, and $[-45,45]$ degrees for steering), the relationship between maximum roll angle and vehicle speed is studied for Vehicle A under different maneuvers.

2. The relationship between allowable hand-wheel steering angle and maximum roll angle is studied for Vehicle A. 
3. Under the same steering angle and brake pedal force limit (200 lbf for pedal force, and for steering, see Table 2), the relationship between surface conditions and maximum roll angle is studied for Vehicle A under different maneuvers.

4. Rollover stability metric comparisons of Vehicle $A$ and Vehicle B are studied.

5. Comparison of maximum hand-wheel steering angles before rollover for Vehicle $A$ and Vehicle $B$, at different vehicle speeds.

\section{PRELIMINARY SIMULATION RESULTS}

Case 1: Our results (Table 1) show that increasing the vehicle speed leads to an increase in maximum roll angle. We see that the worst-case maneuvers found by our method (targeting VSC-off conditions) generate much higher roll angle than the standard maneuvers. It is also clear that the VSC system is effective in reducing the risk of rollover for these worst-case maneuvers since the maximum rollover angle is dramatically decreased in its presence.

Table1: Vehicle speed vs. maximum roll angle for Vehicle A

\begin{tabular}{|c|c|c|c|c|c|}
\hline \multicolumn{3}{|c|}{ speed (kph) } & 80 & 100 & 120 \\
\hline \multirow{2}{*}{$W C^{\prime}$} & VSC-off & \multirow{3}{*}{$\begin{array}{c}\max \\
\text { roll } \\
\text { angle } \\
\text { (deg) }\end{array}$} & 4.22 & 5.53 & rollover \\
\hline & VSC-on & & 1.43 & 1.28 & 3.04 \\
\hline \multicolumn{2}{|c|}{$\begin{array}{l}\text { Standard }^{2} \\
\text { (VSC-off) }\end{array}$} & & 1.57 & 2.18 & 3.03 \\
\hline
\end{tabular}

Simulation conditions: High friction surface for tires; worst-case with respect to steering and braking (WC: Worst-case maneuvers, VSC-on: Worst-case maneuvers with the control system on).

Case 2: Table 2 shows that increasing the hand-wheel steering angle range leads to an increase in maximum roll angle. At each of the steering angle ranges, the maximum roll angle for the worst-case maneuvers is greater than for the standard maneuvers (with steering magnitudes scaled to the same saturation limit). It should be emphasized again that these "worst case" maneuvers are trained to target the VSC-off vehicles. The results found in the row labeled "VSC-on" thus only serve to show that under these three extreme maneuvers, the vehicle equipped with VSC will have much reduced roll angle.

Table2: Allowable hand-wheel steering angle range vs. maximum roll angle for Vehicle $A$.

\begin{tabular}{|c|c|c|c|}
\hline $\begin{array}{c}\text { hand-wheel steering } \\
\text { angle range }(\mathrm{deg})\end{array}$ & {$[-30,30]$} & {$[-45,45]$} & {$[-60,60]$} \\
\hline
\end{tabular}

\footnotetext{
${ }^{1}$ WC maneuver for VSC-off.

${ }^{2}$ Five standard maneuvers: J-Turn with and without pulse braking, fishhook \#1 and \#2, resonant steer maneuver. These maneuvers were used in dynamic testing procedure for the NHTSA Phase II study.
}

\begin{tabular}{|c|c|c|c|c|c|}
\hline \multirow[t]{2}{*}{$\mathrm{WC}^{\prime}$} & VSC-off & \multirow{3}{*}{$\begin{array}{c}\max \\
\text { roll }\end{array}$} & 2.95 & 4.22 & 5.35 \\
\hline & VSC-on & & 1.92 & 1.43 & 2.04 \\
\hline \multicolumn{2}{|c|}{$\begin{array}{l}\text { Standard }{ }^{2} \\
\text { (VSC-off) }\end{array}$} & & 1.03 & 1.57 & 2.17 \\
\hline
\end{tabular}

Simulation conditions: High friction surface; vehicle speed is $80 \mathrm{kph}$; worst-case with respect to steering and braking.

Case 3: Increasing surface friction leads to an increase in maximum roll angle (Table 3 ), and thus elevates the risks for rollover. Similar to the previous cases, worstcase maneuvers are more effective in showing the vehicle's lateral stability under these conditions. On a high friction surface, having the VSC system in Vehicle A protects the vehicle from rollover even when the worstcase maneuvers are applied to the vehicle.

Table3: Surface condition vs. max. roll angle for Vehicle A.

\begin{tabular}{|c|c|c|c|c|}
\hline \multicolumn{3}{|c|}{ surface condition } & low friction & high friction \\
\hline \multirow{2}{*}{ WC $^{1}$} & VSC-off & & 3.24 & rollover $^{3}$ \\
& VSC-on & \multirow{2}{*}{$\begin{array}{c}\text { max roll } \\
\text { angle } \\
\text { (deg) }\end{array}$} & 1.38 & $3.04^{4}$ \\
$\begin{array}{c}\text { Standard } \\
\text { SVS-off) }\end{array}$ & & 2.58 & $3.03^{5}$ \\
\hline
\end{tabular}

Simulation conditions: Vehicle speed is $120 \mathrm{kph}$; hand-wheel steering angle range is $[-45,45]$, worst-case with respect to steering and braking.

Case 4: In this case study, vehicle parameters and steering limits are deliberately selected to make the vehicle less likely to rollover, but nevertheless exhibit reasonably large roll motion. The maximum roll angle is subsequently used as an indication of relative vulnerability to rollover. Under a predefined range of test conditions, multiple "worst-case" results were identified (each of which is a local minimum point in the overall optimization problem). When the uncontrolled Vehicle A and Vehicle B, under their respective worst-case maneuvers, are compared the maximum roll angles of these two SUVs are consistent with what would be obtained using "static" type of metrics. The Static Stability Factors (SSF) for Vehicle A and Vehicle B are 1.03 and 0.96 respectively. These numbers indicate that Vehicle $A$ is expected to be less prone to rollover than Vehicle B. Consistent with this result, our worst-case methodology yields greater maximum roll angles for Vehicle B than for Vehicle A (Fig. 5). This finding is not surprising. However, it should be mentioned that there are plenty of examples in which a "worst-case" maneuver generated by our algorithm that targets the weakness of Vehicle A actually generates higher roll motion on Vehicle A than Vehicle B. An example maneuver is shown in Figure 6. It can be seen that even

\footnotetext{
${ }^{3}$ See appendix.

${ }^{4}$ See appendix.

${ }^{5}$ See appendix.
} 
though the SSF of Vehicle A is $7 \%$ higher, it rolls over at this maneuver while (the supposedly less safe) Vehicle $B$ does not even experience wheel lift-off. In other words, the susceptibility of SUVs to rollovers cannot be concluded from one or a handful of dynamic maneuvers.

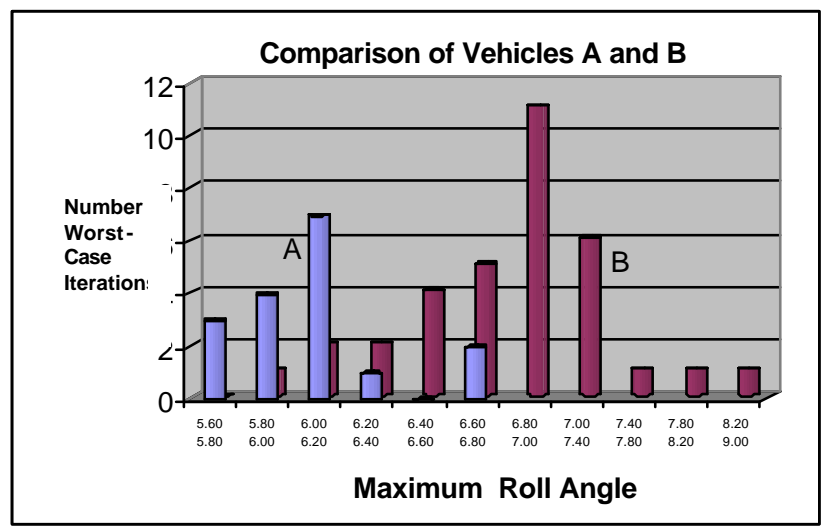

Figure 5: Comparison of maximum roll angles obtained through worst-case evaluation for Vehicle A and Vehicle B. Simulation conditions: Vehicle speed is $100 \mathrm{kph}$; hand-wheel steering angle range is $[-100,100]$ degrees; high friction surface for tires; worst-case with respect to steering and braking; VSC off.

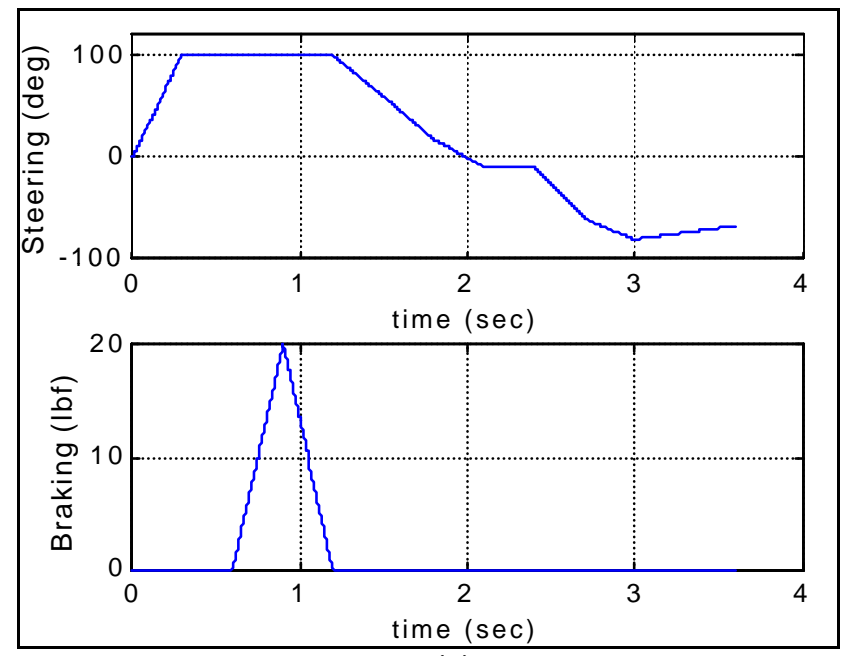

(a)

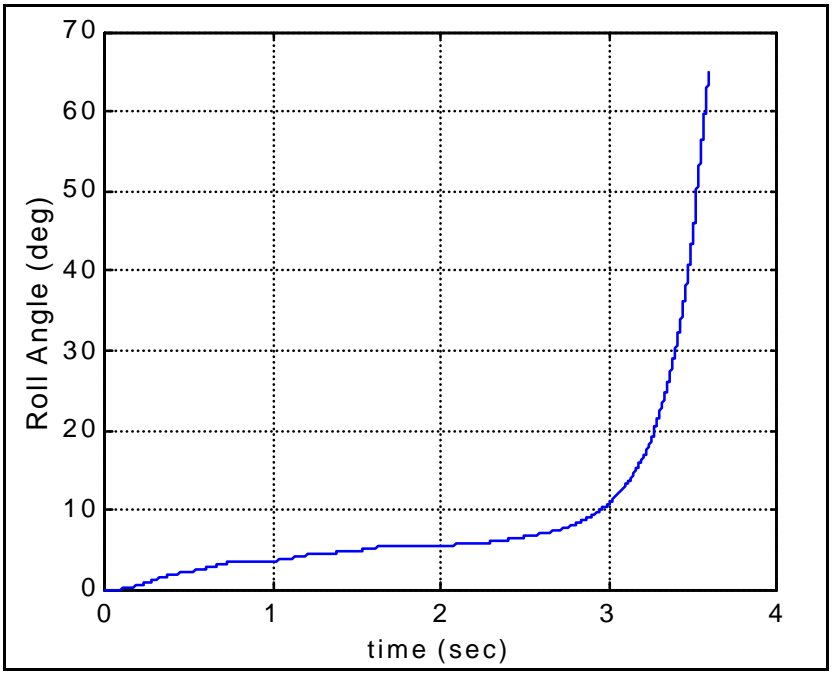

(b)

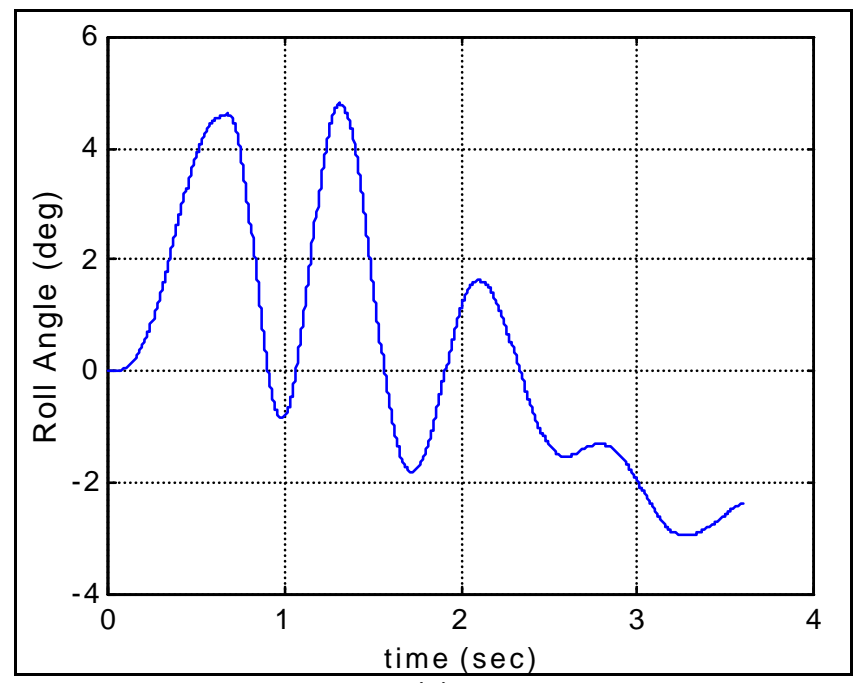

(c)

Figure 6: Example worst case input (a) and the roll response from vehicle A (b) and Vehicle B (c). Initial vehicle speed $=100 \mathrm{kph}$, on high friction surface.

Case 5: In this case study, maximum hand-wheel steering angle values before rollover are compared for the Vehicle A and Vehicle B. Worst-case evaluation method is used to search for the minimum hand-wheel steering angle range that leads to rollover. After achieving rollover for the initial range of the hand-wheel steering angle, range is reduced until rollover was unachievable. This procedure is repeated for the four different values of vehicle speed. It can be seen from figure 7 that up to around $105 \mathrm{kph}$ Vehicle A seems to be harder to roll than Vehicle $B$, whereas at higher speeds relative propensity of rollover for the vehicles reversed, showing that rollover propensity is speed dependent.

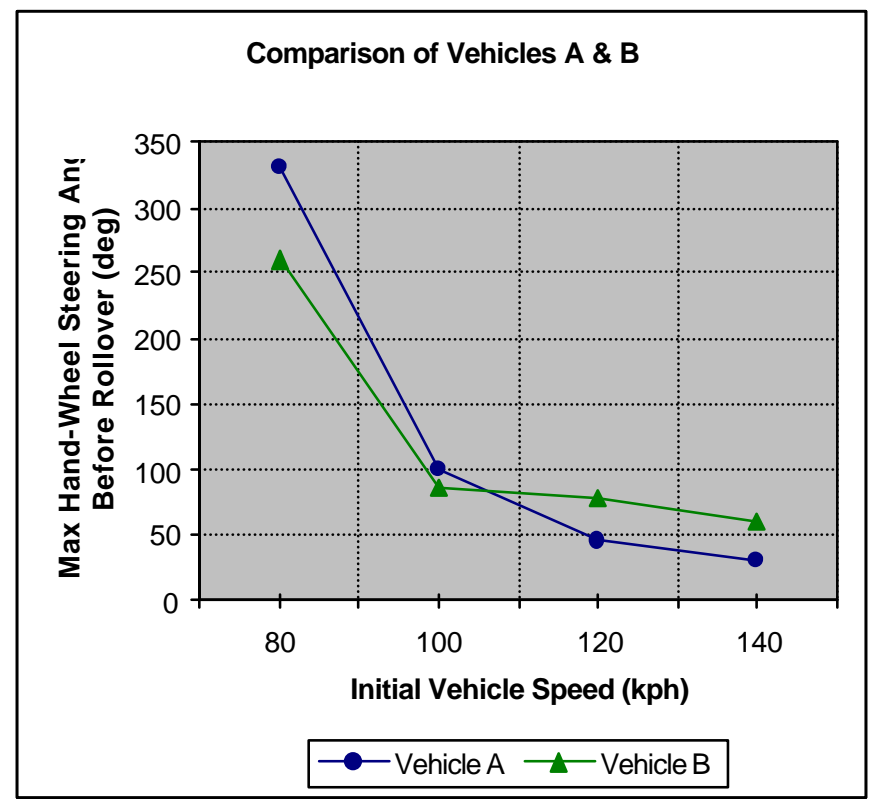

Figure 7: Comparison of Maximum Hand-wheel Steering Angle Values before rollover for the Vehicles A \& B. Simulation conditions: High friction surface; worst-case with respect to steering and braking; VSC off. 


\section{DISCUSSION}

The goals of this paper are to study the effects of vehicle and environment parameters and the influence of VSC systems on the rollover propensity of Sport Utility Vehicles. Five cases were studied in this paper, in the first three cases, worst-case evaluations are performed for different simulation conditions (speed, hand-wheel steering angle and surface friction). Our results show that for all three parameters tested in this study, the maximum roll angle increases along with the magnitude of the parameter. Thus, an increase in the road friction, in the speed or steering range of the vehicle elevates the risks of rollover. For all these cases, VSC was found to reduce the maximum roll angles significantly to about half of the corresponding VSC-off cases. In the fourth case, VEHICLE $A$ and VEHICLE $B$ are compared in terms of their propensity to rollover. The results show that VEHICLE B is more likely to rollover than VEHICLE $A$ as would be expected from the comparison of the SSF values of the two vehicles. In the last simulation case, maximum hand-wheel steering angle values before rollover for Vehicles A and B are compared under various forward speeds. Results show that the rollover propensity is speed dependent..

The results presented in this paper are important since it shows that a VSC system reduces the maximum roll angle by improving the vehicle's lateral stability. Besides, the dynamic rollover evaluation procedure used to determine the worst-case maneuvers for SUVs brings a new dimension to be considered by the government in the establishment of the "star rating" system. Based on previous studies, NHTSA has decided that from the two types of metrics (static and dynamic) available to test a vehicle's tendency to untripped rollover, the dynamic testing method was less advantageous. The dynamic testing procedure used in the NHTSA Phase II study included five maneuvers ( $\mathrm{J}$-Turn with and without pulse braking, fishhook \#1 and \#2, resonant steer maneuver) that were intuitively selected to represent maneuvers preceding rollover accidents. These maneuvers were tested on 12 vehicles including 4 SUVs and the results showed that the dynamic testing system did not predict the rollover tendency of an SUV better than the static testing method, which is based on a very simple physical principle. Moreover, a specific model of an SUV could become prone to rollover in response to a maneuver whose characteristics are not included among the chosen five. Therefore, the dynamic test procedure was determined to be too complex and does not add enough benefit beyond simpler approaches such as SSF.

A better dynamic testing approach to study the vehicle rollover propensity is to first find maneuvers that destabilize the vehicle most effectively. Assessing the risk of the vehicle's rollover under the conditions of these worst-case maneuvers will inform us about the safety of this particular SUV. However, such an approach requires an accurate dynamic model and is computationally intensive. As already stated, speed, surface friction and steering range, the operating parameters studied in this paper, are shown to influence the maximum roll angle for the worst-case maneuvers. Thus, SSF is an insufficient indicator of a vehicle's lateral stability. Moreover, this index also fails to reflect the potential performance of vehicles equipped with a VSC system. As indicated before, for vehicles with a VSC system, the maximum roll angles for worst-case maneuvers are reduced. With a VSC system, it is possible for an SUV with a low SSF to become more stable than one with a higher SSF but no VSC. Therefore, even though SSF is simple and captures the average behavior of vehicles without control systems, it is not the best way to measure the untripped rollover risks of a vehicle. The final case study, and in particular, Figure 6, clearly indicates that it is not easy to assess a vehicle's rollover stability.

The simulation-based dynamic rollover evaluation procedure presented in this paper introduces a better method to measure the rollover risks of SUVs than the static and dynamic testing procedures do. Our method does not have the disadvantages of the "dynamic testing" because it finds the characteristics of the worstcase maneuvers for a vehicle through iterative simulations promptly, and thus is less time consuming. Moreover, this method takes the operating parameters and any control systems the vehicle might have into account while finding the worst-case maneuvers. Therefore, the maximum roll angles found by applying these maneuvers to the vehicles are a more reliable indicator of a vehicle's resistance to rollover than SSF. When we consider NHTSA's intention to use SSF as the more reliable rollover risk indicator, the implications of our results become crucially important. Obviously, our worst-case methodology could be a valuable tool in ranking vehicles for their safety.

\section{CONCLUSIONS}

The results we have obtained in our study have two main messages. First, Vehicle Stability Control system is an important product that can improve vehicle stability against rollovers, especially for SUVs. Because of its implications with respect to consumer safety, this control system constitutes a new issue to be considered. Before the U.S. Congress (and National Academy of Sciences) approves the new "star rating" system and endorses SSF as the indicator of a vehicle's rollover risk, the difference a control system makes in reducing the rollover risk of the vehicle should be considered. Having VSC has a dramatic impact on the roll behavior of Vehicle A. It elevates the vehicle's resistance to rollover. The failure by government agencies to recognize the benefits of these innovative control systems might inadvertently discourage vehicle manufacturers from equipping their vehicles with VSC or other innovative devices. This, in turn, could negatively impact consumer safety. Our worst-case methodology suggests an alternative to existing "dynamic" and "static" types of rollover metrics. Whereas using SSF can be considered to be a better approach than dynamic testing in evaluating the rollover risks, our method introduces a new simulation-based procedure that finds the worst- 
case maneuvers (for rollovers). This method has its advantages compared with SSF mainly because the effect of vehicle parameters, environment parameters and on-board control systems such as VSC are included in its calculations. More importantly, the worst-case method identifies the weak links of vehicles and/or the active control systems, and thus could be a valuable tool in the vehicle design process.

\section{ACKNOWLEDGMENTS}

This research project is supported by TRW Inc. and a grant from the National Science Foundation. The authors also wish to thank M. Sayers and S. Riley of MSC for making TruckSim available for this research.

\section{REFERENCES}

Ėech, I., " Anti-Roll and Active Roll Suspensions ", Vehicle System Dynamics, no 33, pp. 91-106, 2000.

Chen, B. and Peng, H., "A Rollover Warning Algorithm for Sports Utility Vehicles," Proceedings of the 1999 American Control Conference, San Diego, CA.

Constantine, C.J. and Law, A.H., " The Effects of Roll Control for Passenger Cars during Emergency Maneuvers ", SAE Paper No. 940224, 1994.

Furleigh, D.D, Vanderploeg, M.J. and Oh, C.Y., " Multiple Steered Axles for Reducing the Rolloever Risk of Heavy Articulated Trucks ", SAE Paper No. 881866, 1988.

Howe, J. G., Garrott, W. R., Forkenbrock, G., "An Experimental Examination of Selected Maneuvers That May Induce On -Road, Untripped, Light Vehicle Rollover- Phase II of NHTSA's 1997-1998 Vehicle Rollover Research Program", NHTSA Technical Report, July 1999.

Luus, R., " Iterative Dynamic Programming ", Chapman \& Hall/Crc, Monographs and Surveys in Pure and Applied Mathematics, 2000.Ma, W. and Peng, H., "A Worst-Case Evaluation Method for Dynamic Systems," ASME J. of Dynamic Systems, Measurement and Control, Vol.121, No.2, June 1999, pp.191-199.

Palkovics,L., Semsey, A. and Gerum, E., " Roll-Over Prevention Systems for Commercial Vehicles Additional Sensorless Function of the Electronic Brake System," $4^{\text {th }}$ International Symposium on Advanced Vehicle Control, Nagoya, Japan, 1998.

Wielenga, T.J., " A Method for Reducing On-Road Rollovers - Anti-Rollover Braking ", SAE Congress, SAE Paper No. 1999-01-0123, 1999.

\section{APPENDIX}
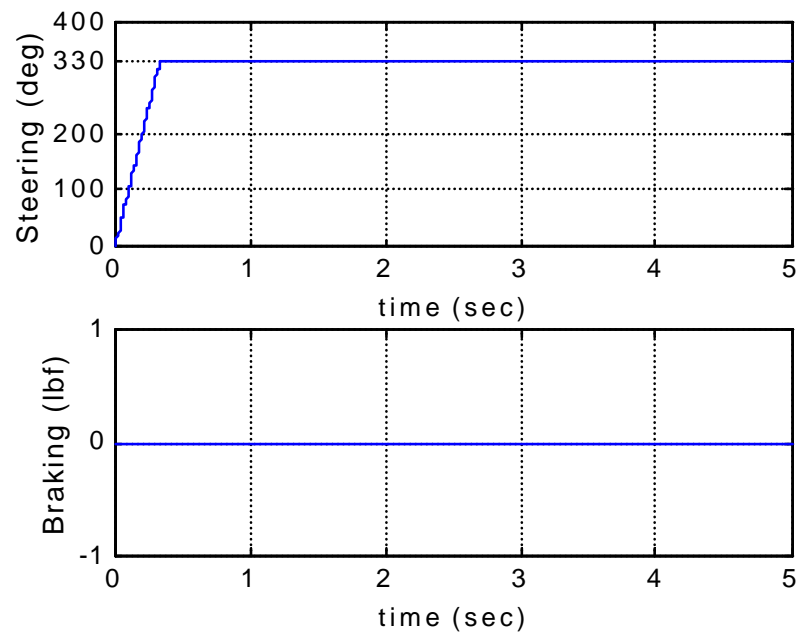

Figure A1: J-Turn (NHTSA Tech. Report 1999).
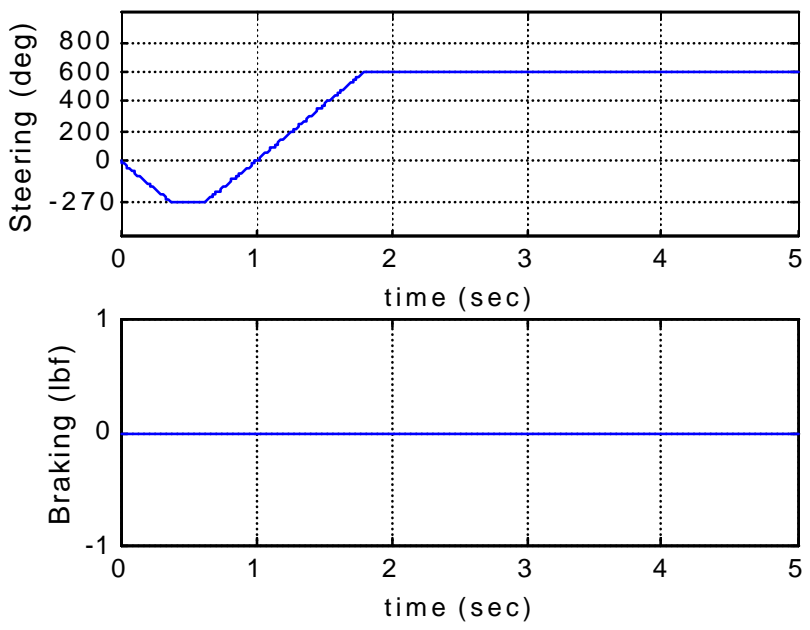

Figure A2: Fishhook \#1(NHTSA Tech. Report 1999).
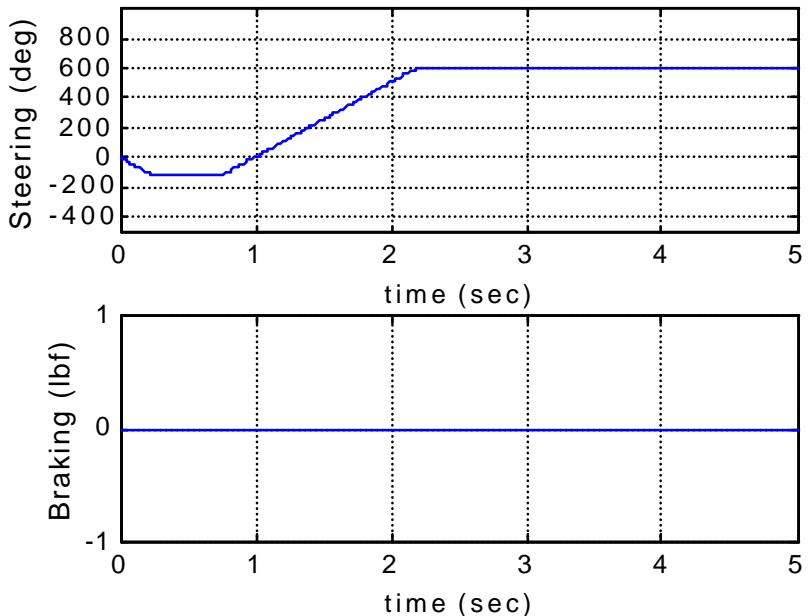

Figure A3: Fishhook \#2(NHTSA Tech. Report 1999). 

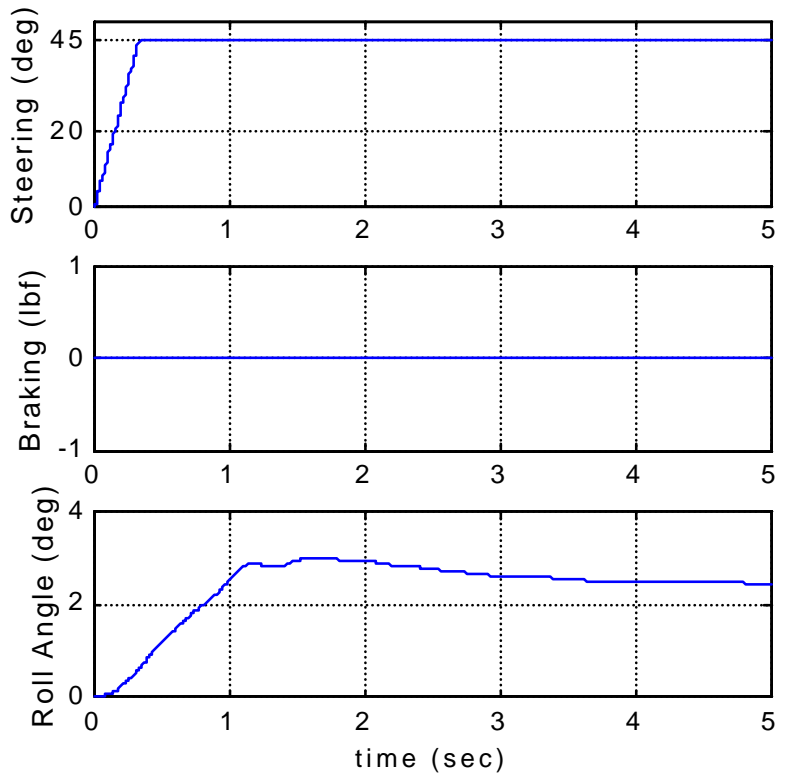

Figure A4: J-Turn scaled to $[-45,45]$ deg. steering angle range. (steering as defined in figure $A 1 * 45 / 330$ ). Example roll angle obtained with vehicle speed $=120 \mathrm{kph}$, high friction surface, VSC-off.
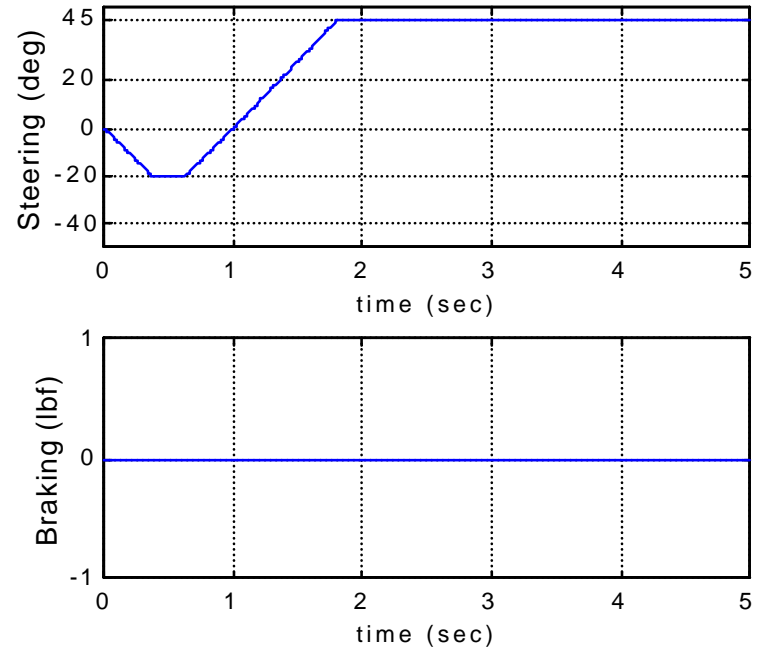

Figure A5: Fishhook \#1 scaled to $[-45,45]$ deg. steering angle range.
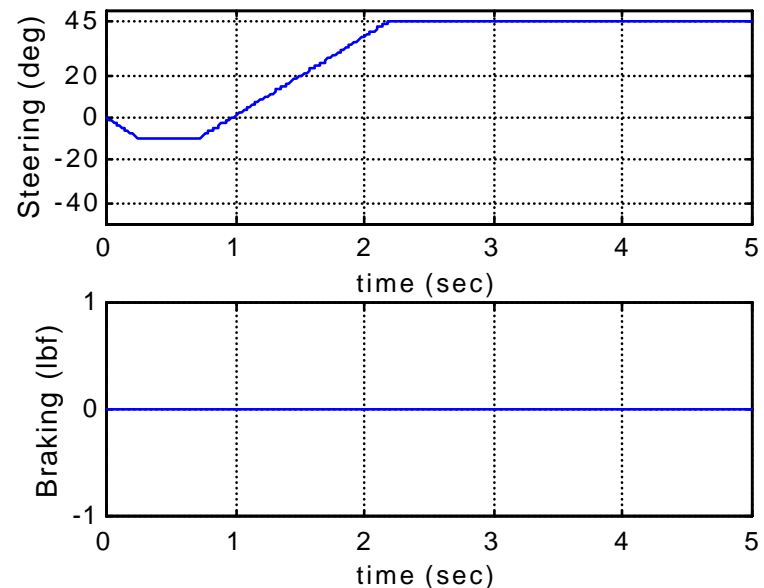

Figure A6: Fishhook \#2 scaled to $[-45,45]$ deg. steering angle range.
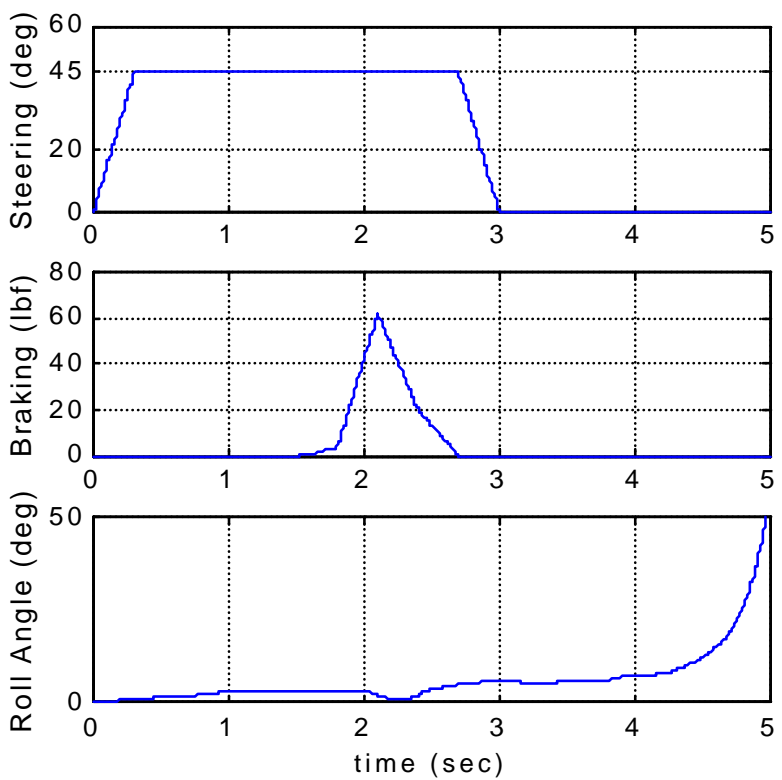

Figure A7: Vehicle speed is $120 \mathrm{kph}$; hand-wheel steering angle range is $[-45,45]$, worst-case with respect to steering and
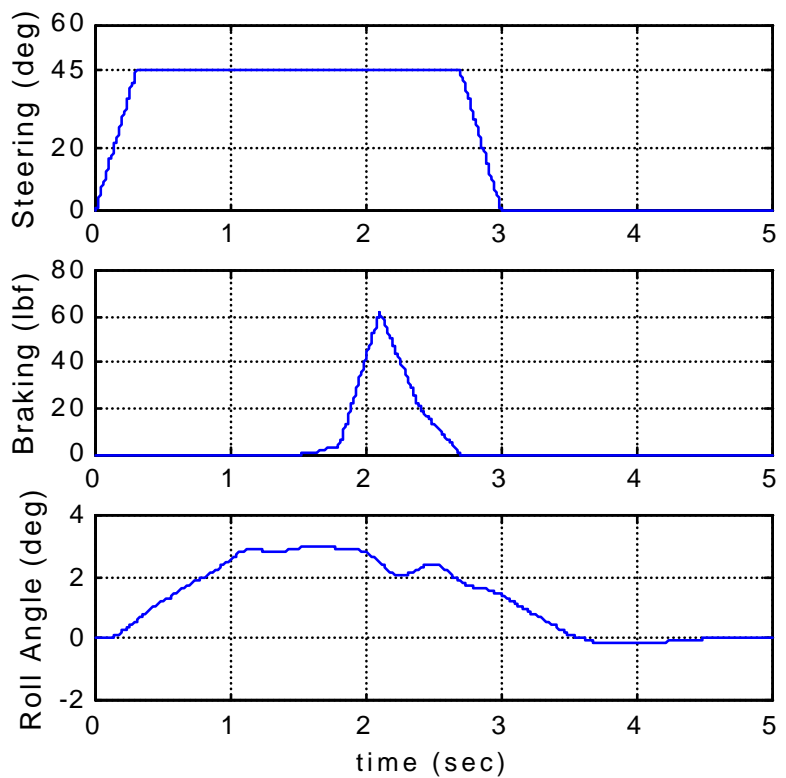

Figure A8: Vehicle speed is $120 \mathrm{kph}$; hand-wheel steering angle range is $[-45,45]$, worst-case with respect to steering and braking, high friction surface, VSC-on. 